\title{
Author Correction: Genome-wide mapping of plasma protein QTLs identifies putatively causal genes and pathways for cardiovascular disease
}

\author{
Chen Yao 10 1,2, George Chen 1,2, Ci Song1,2,3,4, Joshua Keefe ${ }^{1,2}$, Michael Mendelson 1,2,5, Tianxiao Huan ${ }^{1,2}$, \\ Benjamin B. Sun (10 6 , Annika Laser ${ }^{7,8}$, Joseph C. Maranville9, Hongsheng Wu ${ }^{10}$, Jennifer E. Ho ${ }^{11}$, \\ Paul Courchesne 1,2, Asya Lyass 1,12, Martin G. Larson1,13, Christian Gieger7,8,14, Johannes Graumann (i) ${ }^{15}$, \\ Andrew D. Johnson 1,2, John Danesh6,16,17, Heiko Runz ${ }^{9}$, Shih-Jen Hwang ${ }^{1,2}$, Chunyu Liu ${ }^{1,2}$, \\ Adam S. Butterworth (i) ${ }^{6,18}$, Karsten Suhre (iD ${ }^{19}$ \& Daniel Levy ${ }^{1,2}$
}

Correction to: Nature Communications; https://doi.org/10.1038/s41467-018-05512-x; published online 15 August 2018.

In the originally published version of this article, financial support was not fully acknowledged. The sentence "KS was supported by the 'Biomedical Research Program' funds at Weill Cornell Medicine in Qatar, a program funded by the Qatar Foundation” has been added to the acknowledgement section in both the PDF and HTML versions of the article.

Published online: 18 September 2018

\begin{abstract}
(c) Open Access This article is licensed under a Creative Commons Attribution 4.0 International License, which permits use, sharing, adaptation, distribution and reproduction in any medium or format, as long as you give appropriate credit to the original author(s) and the source, provide a link to the Creative Commons license, and indicate if changes were made. The images or other third party material in this article are included in the article's Creative Commons license, unless indicated otherwise in a credit line to the material. If material is not included in the article's Creative Commons license and your intended use is not permitted by statutory regulation or exceeds the permitted use, you will need to obtain permission directly from the copyright holder. To view a copy of this license, visit http://creativecommons.org/licenses/by/4.0/.
\end{abstract}

(C) The Author(s) 2018

\footnotetext{
${ }^{1}$ Framingham Heart Study, Framingham 01702, MA, USA. ${ }^{2}$ Population Sciences Branch, Division of Intramural Research, National Heart, Lung, and Blood Institute, National Institutes of Health, Bethesda 20892, MD, USA. ${ }^{3}$ Department of Medical Sciences, Uppsala University, 75105 Uppsala, Sweden. ${ }^{4}$ Department of Immunology, Genetics and Pathology, Uppsala University, 75105 Uppsala, Sweden. ${ }^{5}$ Department of Cardiology, Boston Children's Hospital, Boston 02115, MA, USA. ${ }^{6}$ MRC/BHF Cardiovascular Epidemiology Unit, Department of Public Health and Primary Care, University of Cambridge, Cambridge CB1 8RN, UK. ${ }^{7}$ Research Unit of Molecular Epidemiology, Helmholtz Zentrum München, German Research Center for Environmental Health, Ingolstädter Landstraße 1, 85764 Neuherberg, Germany. ${ }^{8}$ Institute of Epidemiology II, Helmholtz Zentrum München, German Research Center for Environmental Health, Ingolstädter Landstraße 1, 85764 Neuherberg, Germany. ${ }^{9}$ MRL, Merck \& Co., Inc, Kenilworth 07033, NJ, USA. ${ }^{10}$ Computer Science and Networking, Wentworth Institute of Technology, Boston 02115, MA, USA. ${ }^{11}$ Cardiovascular Research Center and Division of Cardiology, Department of Medicine, Massachusetts General Hospital, Boston 02114, MA, USA. ${ }^{12}$ Department of Mathematics and Statistics, Boston University, Boston 02115, MA, USA. ${ }^{13}$ Department of Biostatistics, Boston University School of Public Health, Boston 02118, MA, USA. ${ }^{14}$ German Center for Diabetes Research (DZD), Ingolstädter Landstraße 1, 85764 Neuherberg, Germany. ${ }^{15}$ Scientific Service Group Biomolecular Mass Spectrometry, Max Planck Institute for Heart and Lung Research, W.G. Kerckhoff Institute, Ludwigstr. 43, D-61231 Bad Nauheim, Germany. ${ }^{16}$ British Heart Foundation Cambridge Centre of Excellence, Division of Cardiovascular Medicine, Addenbrooke's Hospital, Cambridge CB2 OQQ, UK. ${ }^{17}$ Department of Human Genetics, Wellcome Trust Sanger Institute, Wellcome Trust Genome Campus, Hinxton, Cambridge CB10 1RQ, UK. ${ }^{18}$ NIHR Blood and Transplant Research Unit in Donor Health and Genomics, Department of Public Health and Primary Care, University of Cambridge, Cambridge CB1 8RN, UK. ${ }^{19}$ Department of Physiology and Biophysics, Weill Cornell Medicine-Qatar, Education City, PO 24144 Doha, Qatar. Correspondence and requests for materials should be addressed to D.L. (email: levyd@nih.gov)
} 